\title{
USO DEL CAUCHO DE NEUMÁTICOS TRITURADOS Y APLICADOS AL CONCRETO: UNA REVISIÓN LITERARIA
}

\author{
USE OF RUBBER IN TIRES CRUSHED AND APPLIED TO CONCRETE: A \\ LITERARY REVIEW
}

\author{
Sócrates Muñoz; José Vidaurre; James Asenjo; Roberth Gavidia \\ Universidad Señor de Sipán. Campus Universitario Carretera a Pimentel km 5, Chiclayo - Perú.
}

Email:msocrates@crece.uss.edu.pe

https://doi.org/10.33789/talentos.8.1.142

\begin{abstract}
Resumen: Los avances en la tecnología del concreto conducen a la búsqueda de materiales alternativos que aporten mejoras en las propiedades del concreto y al mismo tiempo contribuyan con reducir la contaminación del medio ambiente. En este artículo se revisan resultados de investigaciones reportados en 50 artículos indexadas en la base de datos Scopus durante los años 2017 al 2020, y que tenían como objetivo estudiar las propiedades del concreto con inclusiones de caucho proveniente de neumáticos reciclados. Se revisan las metodologías y técnicas de procesamiento del caucho para darle la forma y el tamaño requerido, así como también los resultados alcanzados con la incorporación del caucho en el concreto. En conclusión, se discutirán las recomendaciones para las futuras investigaciones y la aplicación del caucho de neumático en el concreto.
\end{abstract}

Palabras Clave: Trituración; Caucho; Concreto; Propiedades físicas.

\begin{abstract}
Advances in concrete technology lead to the search for alternative materials that provide improvements in concrete properties and at the same time contribute to reducing environmental pollution. This article reviews research results reported in 50 articles indexed in the Scopus database during the years 2017 to 2020, and which aimed to study the properties of concrete with rubber inclusions from recycled tires. Rubber processing methodologies and techniques are reviewed to give it the required shape and size, as well as the results achieved with the incorporation of rubber into concrete. In conclusion, recommendations for future research and application of tire rubber in concrete will be discussed.
\end{abstract}

Recibido: 15 de diciembre de 2020

Aceptado: 30 de marzo de 2021

Publicado como review en la Revista de Investigación Talentos VIII (1), 36-51 
Keywords: Trituration; Rubber; Concrete; Physical properties.

\section{INTRODUCCIÓN}

La cantidad de neumáticos desechados a nivel mundial durante las últimas décadas ha generado un gran interés y preocupación sobre su impacto en la salud de los humanos y del ambiente (Youssf, y otros, 2020). Se estima que aproximadamente 1,500 millones de neumáticos de desecho se generan cada año en todo el mundo (XU, Yao, Yang, \& Han, 2020). El caucho se puede usar como reemplazo tanto de agregado fino como de agregado grueso en la producción de concreto (Senin, y otros, 2017), dado que el neumático de desecho no es biodegradable y tiene el potencial de causar problemas ambientales (Si, Guo, \& Dai, 2017). La incorporación del caucho en el concreto puede ser una de las posibles soluciones para el aprovechamiento de los neumáticos desechados y la conservación de los recursos naturales (Al Rawahi \& Bilal Waris, 2017). Con el creciente interés en la construcción sostenible y respetuosa con el medio ambiente, el concreto con agregados de material reciclado se ha investigado ampliamente y se está incorporando gradualmente en proyectos de ingeniería (Lei, Liu, Yao, \& Tang, 2019). Los neumáticos desechados pueden reciclarse en diversas aplicaciones en la industria de la construcción, por ejemplo, en la producción de concreto, como agregado en: obras estructurales, pavimentos de carreteras, estructuras antisísmicas, muros de contención y estuarios, entre otros (Bisht \& Ramana, 2017). Por lo tanto, existe la necesidad de un mayor conocimiento sobre el efecto que tiene la incorporación de caucho en el desempeño del concreto (Roychand et al., 2019). Hasta la fecha, se han realizado una gran cantidad de trabajos de investigación para determinar los efectos de partículas de caucho en las propiedades del concreto. Así como en las diversas formas de procesamiento del neumático para lograr los agregados de caucho que se desean y utilizarlas en el concreto ( $\mathrm{Lv}, \mathrm{Du}$, Zhou, $\mathrm{He}, \& \mathrm{Li}, 2019)$. Las investigaciones más relevantes se concentran en el uso del caucho provenientes de neumáticos reciclados con la finalidad de estudiar la resistencia normal del concreto, pero hay pocos estudios disponibles que analicen el efecto de agregar caucho en el concreto de alta resistencia (Abdelmonem, El-Feky M, Nasr, \& Kohail, 2019),así como pocos estudios sobre modelado y predicción de la resistencia a la compresión, las regresiones multivariables no lineales no se han utilizado hasta ahora para la predicción del comportamiento del concreto con caucho (Jalal, Nassir, Jalal, \& Arabali, 2019). Su uso práctico como nuevo material en la construcción requiere de una comprensión profunda y una predicción confiable de sus efectos (Svoboda, Václavík, Dvorský, Klus, \& Botula, 2020). Por lo tanto, una revisión completa actualizada sobre el concreto con caucho de neumáticos reciclados es necesaria para comprender el comportamiento de este importante material de construcción, aunque se han realizado considerables investigaciones, muchas siguen siendo controvertidas (Li, Zhang, Wang, \& Dang, 2019). El objetivo de este artículo es revisar las investigaciones más recientes sobre el uso del caucho de neumáticos reciclados en el concreto. Además, los resultados de esta revisión se analizan y discuten ya que nos pueden proporcionar nuevos conocimientos e información sobre las aplicaciones del caucho sobre el concreto. 


\section{DESARROLLO}

\subsection{METODOLOGÍA}

La revisión se realizó empleando 50 artículos indexados en la base de datos Scopus, donde se encontró, 8 artículos en el año 2017, 4 en el año 2018, 12 en el año 2019 y 26 en el año 2020. Para la búsqueda de los artículos se usaron las siguientes palabras claves: trituración; caucho; concreto; propiedades físicas, trituration; rubber; concrete; physical properties. Se incluyeron todos los artículos sobre estudios de concreto estructural y se excluyeron artículos con estudios de concreto asfaltico. Para un mejor detalle en la tabla 1 se muestra los artículos distribuidos según la base de datos y año de publicación.

Tabla 1: Artículos distribuidos según la base de datos y año de publicación

\begin{tabular}{|l|l|l|l|l|l|}
\hline \multirow{2}{*}{$\begin{array}{l}\text { Base de } \\
\text { datos }\end{array}$} & \multicolumn{4}{|c|}{ Año de publicación } & \multirow{2}{*}{ Total } \\
\cline { 2 - 5 } & 2017 & 2018 & 2019 & 2020 & \\
\hline Scopus & 8 & 4 & 12 & 26 & 50 \\
\hline Total & 8 & 4 & 12 & 26 & 50 \\
\hline
\end{tabular}

Fuente: Elaboración propia

\section{RESULTADOS Y DISCUSIÓN}

\section{1.- Tipos de agregados de caucho}

Los neumáticos reciclados se pueden dividir en dos grandes grupos: neumáticos de automóvil y neumáticos de camión, y son diferentes entre sí, por lo que es importante señalar que ambos varían según su forma, peso y tamaño (Gerges, Issa, \& Fawaz, 2018). Los neumáticos reciclados son procesados para usarse como caucho en el concreto y se clasifican en tres categorías:

a) Caucho astillado, utilizado como agregado grueso, es producido en un proceso de dos etapas en el que la etapa primaria tritura el caucho del neumático a un tamaño de 300$460 \mathrm{~mm}$ de largo por 100-230 $\mathrm{mm}$ de ancho, seguido de la etapa secundaria que produce tamaños de partículas que oscilan entre 13 mm y 76 mm (Roychand et al., 2019).

b) Caucho molido, usado como agregado fino, es producido por dos métodos: (a) a temperatura ambiente utilizando molinos de craqueo y (b) a temperaturas inferiores a $80^{\circ} \mathrm{C}$ usando nitrógeno mediante un proceso criogénico, para producir tamaños de partículas que van desde $4,75 \mathrm{~mm}$ a menor a 0,075 mm (Roychand et al., 2019).

c) Caucho molido fino es utilizado como agregado muy fino, el cual sus partículas son de tamaño que varía desde $0,5 \mathrm{~mm}$ hasta tan solo $0,075 \mathrm{~mm}$, producido mediante un proceso de micro fresado o también llamado molienda en húmedo (Roychand et al., 2019)

Cuando el tamaño y el contenido de las partículas de caucho son apropiados, el concreto con caucho puede lograr muchas propiedades excelentes (Yang, Chen, Xuan, \& Chen, 2018). El caucho astillado se comercializa y se utiliza como agregado grueso en yesos, morteros, concreto y asfaltos (Medina, Medina, Hernández-Olivares, \& Navacerrada, 2017). En comparación con el caucho molido y el caucho astillado, la gran mayoría de investigadores utilizan el caucho molido (alrededor de 0.075 a $4.75 \mathrm{~mm}$ ), para reemplazar la arena natural, mientras que el caucho molido fino se puede utilizar como relleno en concreto debido a su tamaño.

\section{2.- Procesamiento de los neumáticos para lograr agregados de caucho.}

Para lograr agregados de caucho de los neumáticos, existen dos procesos: 1) molienda mecánica a temperatura ambiente(piezas pequeñas) y 2) molienda criogénica a una temperatura por debajo de la transición vítrea(piezas diminutas), la etapa empieza 
con la eliminación de fibras de acero, luego la etapa de cribado y molienda y finalmente la etapa de limpieza .En la etapa de limpieza, las partículas de caucho se limpian a fondo con agua y otros agentes de limpieza como solución de amoniaco, acido de cítrico, etc (Mohajerani et al., 2020). ambiente consiste en el pulido mecánico de los neumáticos desechados, sin el control intencional de la temperatura de procesamiento, para formar productos, como cortes, pedazos y astillas de neumáticos de desecho (Mohajerani et al., 2020), ver figura 1 .

\section{La molienda mecánica a temperatura}

Figura 1: Representación esquemática de la molienda ambiental (Mohajerani et al., 2020).

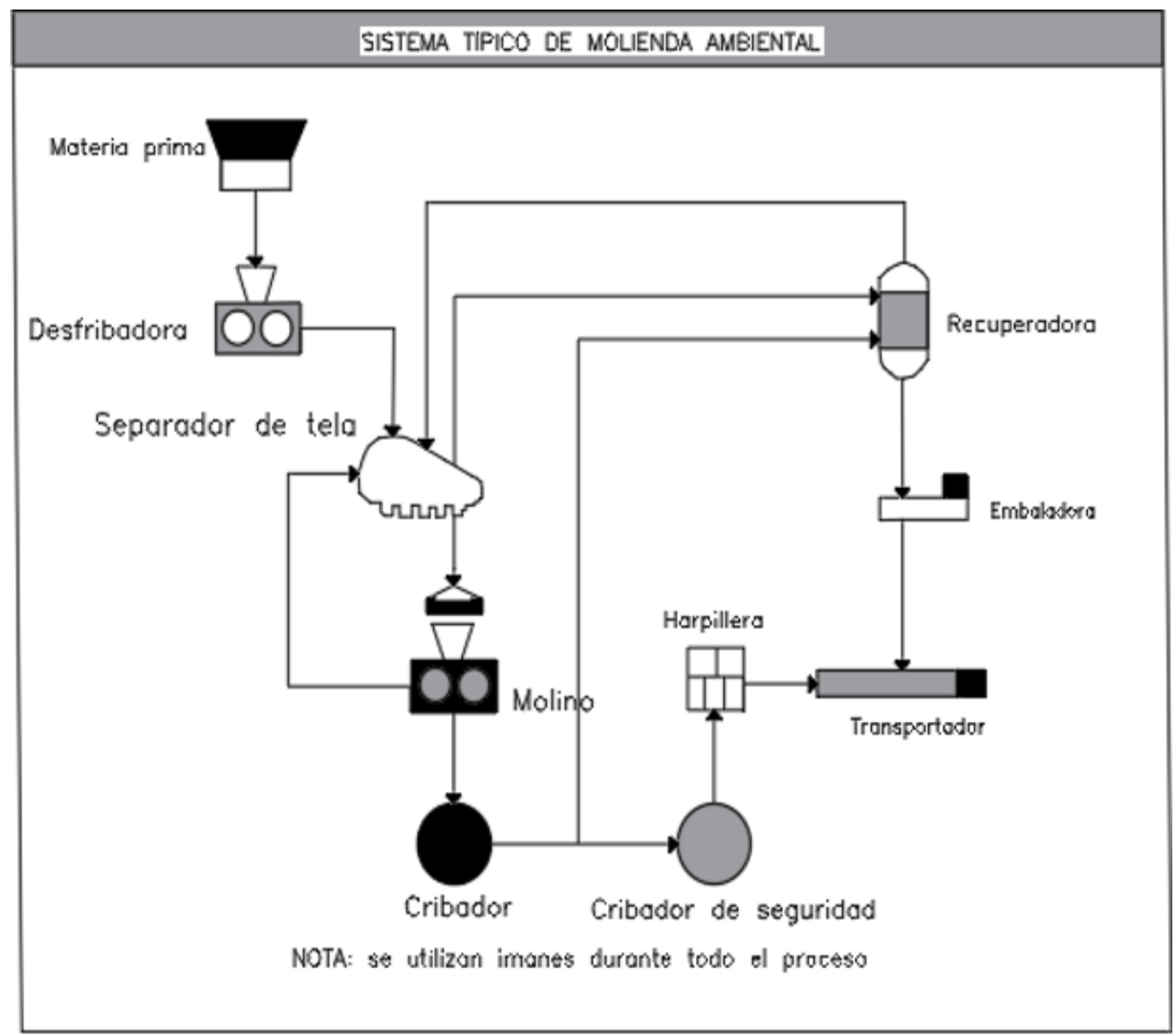

Fuente: Mohajerani et al., 2020.

La molienda criogénica consiste en congelar el caucho con un agente químico, como nitrógeno líquido, por debajo de la fase de transición vítrea. A continuación, se tensiona el caucho hasta obtener el tamaño deseado.
La molienda criogénica produce materiales de caucho más suaves y más pequeñas (Mohajerani, y otros, 2020), ver figura 2. 
Figura 2: Representación esquemática de la molienda criogénica (Mohajerani et al., 2020).

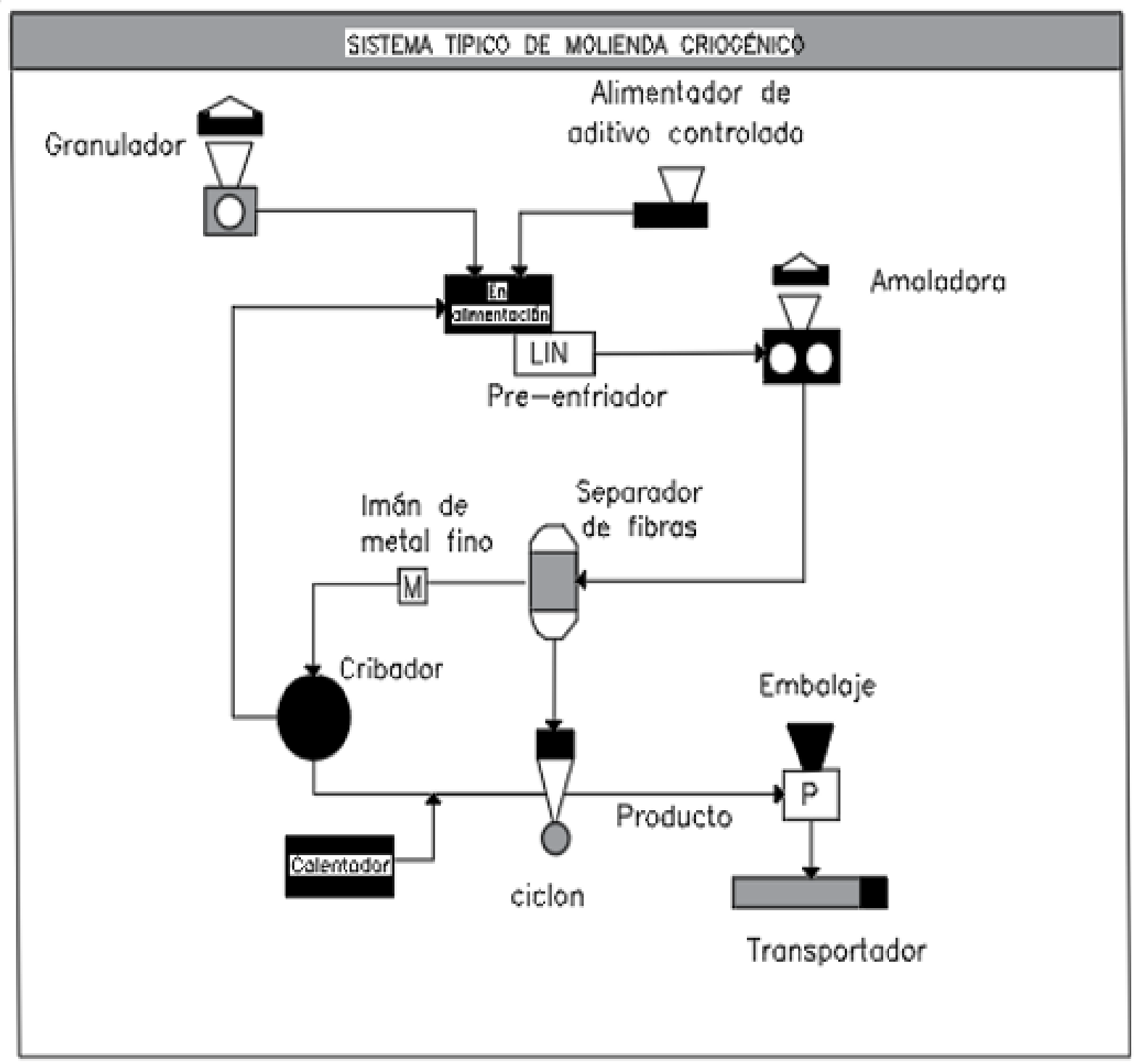

Fuente: Mohajerani et al., 2020.

\section{3.- Costo de procesamiento}

El costo de procesamiento de los neumáticos desechados involucra mano de obra, energía, equipo y su mantenimiento. Por lo tanto, si el tamaño de las partículas de caucho es menor, entonces el costo asociado con su producción será mucho mayor (Roychand et al., 2019). Ver tabla 2.

La diferencia entre procesamiento a temperatura ambiental y criogénica está en la calidad del polvo de caucho. El procesamiento criogénico conduce a una mayor finura de molienda, cuyo precio es mayor (Chylík, Trtík, Fládr, \& Bílý, 2017).
Tabla 2: Costo de procesamiento del caucho

\begin{tabular}{|l|l|l|}
\hline $\begin{array}{l}\text { Tamaño de la } \\
\text { partícula }\end{array}$ & $\begin{array}{l}\text { Costo por } \\
\text { tonelada }\end{array}$ & $\begin{array}{l}\text { Tasa de } \\
\text { procesa mien to } \\
\text { toneladas / hora) }\end{array}$ \\
\hline$>5 \mathrm{~cm}$ & $\$ 12$ & $10-12$ \\
\hline$<5 \mathrm{~cm}$ & $\$ 31$ & 7 \\
\hline$<1.25 \mathrm{~cm}$ & $\$ 31-\$ 68$ & $2-3$ \\
\hline
\end{tabular}

Tabla 2: Costos potenciales de procesamiento y trituración de neumáticos en Canadá (Roychand et al., 2019).

3.4.- Propiedades del concreto que son afectados con la incorporación de agregados de caucho 


\subsubsection{Propiedades mecánicas:}

Hay muchas investigaciones sobre los efectos de las partículas de caucho en las propiedades mecánicas del concreto. La mayoría de las investigaciones se han concentrado en el uso de partículas de caucho redondas conocidas como caucho en migajas que reemplazan parcialmente los agregados finos y / o gruesos en el concreto. También hay algunas investigaciones sobre partículas de caucho de gran tamaño que reemplazaron parcialmente a los agregados gruesos. Además, se han utilizado partículas de caucho muy finas en forma de polvo como relleno en el concreto (Bandarage \& Sadeghian, 2019).

\section{a) Resistencia a la compresión}

Las investigaciones han demostrado que la presencia de agregados con caucho para neumáticos en el concreto altera el desempeño del concreto, tanto positiva como negativamente. Uno de los principales efectos positivos al usar caucho desmenuzado es obtener concretos de alta resistencia con proporciones de 0 a $12.5 \%$, pero su exceso causaría efectos negativos en la resistencia del concreto, la mayoría de los investigadores han sugerido un contenido máximo de caucho de no más del $20 \%$ del volumen total de agregado y un tamaño no mayor que el tamaño del caucho desmenuzado. Se imaginó que el caucho blando actuaba como huecos de aire dentro del concreto, ofreciendo baja resistencia a las cargas, lo que hace que el sitio de las partículas sea un punto débil en la matriz del concreto (Mushunje, Otieno, \& Ballim, 2018).

(Irmawaty, Parung, \& Md Noor, 2020), utilizaron dos tipos de partículas de caucho (cauchodesmenuzadoyvirutas deneumáticos) en las mezclas de concreto con caucho que reemplazan los agregados parcialmente naturales. Los resultados mostraron que el peso del volumen de concreto disminuyó en un 3,5\% en cada adición de un $10 \%$ de caucho desmenuzado + astillas de neumático La disminución en la resistencia a la compresión fue del $24 \%$ con la adición de hasta un $10 \%$ de caucho desmenuzado + virutas de neumáticos, mientras que la resistencia a la tracción dividida disminuyó en un $16 \%$.

(Eltayeb, Ma, Zhuge, Youssf, \& Mills, 2020), investigaron las propiedades frescas $\mathrm{y}$ endurecidas del concreto celular que incorpora caucho fino como reemplazo parcial de la arena de concreto por volumen. Los resultados mostraron que el concreto con caucho con resistencia a la compresión superior a $10 \mathrm{MPa}$ y densidad inferior a 1600 $\mathrm{kg} / \mathrm{m}^{3}$ podría lograrse utilizando un $17 \%$ de contenido de caucho del volumen total de concreto.

Según (Adeboje, Kupolati, Sadiku, \& Ndambuki, 2020), evaluó los efectos de la sustitución parcial de arena en 1,2,3 y 4\% por caucho granulado sobre las propiedades mecánicas y la formación microestructural del concreto de caucho granulado modificado. Se produjo una mejora en la resistencia con 18.5, $21,28.5,41$ y $42.5 \mathrm{MPa}$ y densificación de la microestructura de las muestras de concreto con 2330 y $2340 \mathrm{~kg} / \mathrm{m} 3$ a medida que la edad de curado aumentó de 3 a 7, 28, 90 y 120 días, respectivamente.

(BušiC, BenšiC, MiliceviC, \& Strukar, 2020), investigaron la influencia del caucho de neumáticos desechados y el humo de sílice en las propiedades frescas y endurecidas del concreto fluido (SCC) y diseñar modelos de regresión multivariante para la predicción de las propiedades mecánicas del concreto con caucho autocompactante (SCRC). De acuerdo con los resultados obtenidos, las mezclas con hasta un $15 \%$ de caucho reciclado y un $5 \%$ de humo de sílice, con una resistencia a la compresión de 28 días por encima de 30 $\mathrm{MPa}$, resultaron ser mezclas óptimas para la potencial investigación futura de concreto fluido estructural de caucho. 
(Muttashar, Alomari, \& Al-Umar, 2020), exploraron el efecto de reemplazar el agregado grueso con caucho de neumáticos desechados de acuerdo con el tamaño de los tamices. Se sugirieron tres proporciones de reemplazo para sustituir el agregado grueso en el concreto de $5 \%, 10 \%$ y $15 \%$ en peso. Los resultados ilustraron que la resistencia a la compresión se redujo de $51 \%$ a $15 \%$ reemplazando todos los tamaños de partículas.

(Silva et al., 2019), investigaron la influencia de la incorporación del caucho proveniente de neumáticos desechados en las propiedades mecánicas del concreto de alto rendimiento. El caucho residual del proceso de recauchutado de neumáticos se utilizó en sustitución parcial del agregado fino en los porcentajes de 7,5\%, $15 \%$ y $30 \%$ con respecto a la masa de la arena. Los resultados muestran que con el aumento de la sustitución de la arena por caucho, hubo una disminución de la resistencia mecánica: para la resistencia compresiva a los 28 días, la disminución en relación con el concreto sin caucho fue del $21,8 \%, 36,7 \%$ y $51,7 \%$, respectivamente, para el $7,5 \%, 15 \%$ y $30 \%$ de reemplazo; para la resistencia a la tracción en la flexión, no disminuyó con el 7,5\% de la sustitución y para el $15 \%$ y el $30 \%$ el descenso fue, respectivamente, del 17,7\% y del 48,9\%; para el módulo de elasticidad el descenso fue del $1,5 \%, 12,9 \%$ y $32,5 \%$.

(Huang, Huang, Xing, \& Zhou, 2020), investigaron la relación entre el grado equivalente de compactación (EDC) y el factor de reducción de resistencia (SRF) del concreto de caucho granulado. Se observó una estrecha correlación entre el SRF y el EDC, y puede explicar que los agregados de caucho en el concreto pueden considerarse como huecos regulares. Sobre la base de la teoría de microporosidad, se desarrolló un nuevo modelo SRF de concreto de caucho granulado.

(Najmi, Mariyana, Shek, \& Nurizaty, 2020), investigaron las propiedades de endurecimiento del concreto puzolánico engomado (RuPC) con diferente proporción de caucho en migajas y cenizas volantes como agregado fino parcial y reemplazo del cemento Portland ordinario (OPC), respectivamente. El contenido de caucho en migajas que reemplaza el agregado fino está en el rango de $0 \%$ a $20 \%$, mientras que las cenizas volantes que reemplazan al cemento varían de $0 \%$ a $30 \%$. La resistencia general de RuPC disminuye a medida que aumenta el contenido de caucho en polvo, en el que el $5 \%$ de caucho en polvo muestra la menor reducción de resistencia.

(Shao et al., 2020), realizaron experimentos de compresión, flexión, derrame, deflexión y deformación en concreto con partículas de caucho finas, medias y gruesas añadidas respectivamente al 5\%,10\%, 15\%, 20\% y $25 \%$. concluyeron que mientras se mantiene una resistencia interfacial adecuada necesaria como material de reparación, el caucho en el concreto ayuda a aumentar la deformabilidad, lo cual es beneficioso para compensar la rigidez del concreto.

(Banerjee, Mandal, \& Dr. Jessy Rooby, 2020), en su estudio usaron el polvo de caucho en reemplazo del agregado fino por diferentes porcentajes Los experimentos a realizar fue reemplazar arena (agregado fino) por polvo de caucho en $0 \%, 5 \%, 7 \%, 10 \%$ y verificar la resistencia a la compresión en diferentes condiciones.

\section{b) Resistencia a la flexión}

(Choudhary, Chaudhary, Jain, \& Gupta, 2020), usaron fibras de caucho como sustituto de agregados finos. Utilizaron diferentes materiales como cenizas volantes, fibras de acero, gránulos de vidrio, agregados de granito triturado, etc. para preparar concreto funcionalmente graduado. El porcentaje de reemplazo de fibras de caucho fue del 0 al 20\% a intervalos del 5\% y del 30\% para preparar 
concreto de control, concreto de fibra de caucho y concreto con caucho funcionalmente clasificado. El estudio concluyó que concreto con caucho podría ser un enfoque sostenible hacia la construcción donde actúa una mayor resistencia a la flexión.

\section{c) Resistencia a la tensión}

(Mousavimehr \& Nematzadeh, 2019), investigaron el comportamiento de tensión - deformación residual del concreto que contiene caucho como reemplazo natural de la arena (en 0,15 y 30\%) bajo compresión axial después de la exposición a temperaturas elevadas $\left(200,400,600\right.$ y $\left.800{ }^{\circ} \mathrm{C}\right)$. Para ello, se examinaron las propiedades físicas y mecánicas de las probetas de concreto, incluidas la resistencia a la compresión, tracción, módulo de elasticidad, deformación en la tensión máxima, deformación máxima, tenacidad, curva de tensión-deformación y la pérdida de peso, así como sus características visuales exposición, y luego se propusieron varios modelos empíricos para la predicción de propiedades mecánicas.

\section{d) Resistencia al agrietamiento}

(Sun, Wu, Yang, \& Yang, 2020), evaluaron el efecto potencial del caucho en migajas provenientes de neumáticos desechados sobre las propiedades mecánicas y la resistencia al agrietamiento del macadán estabilizado con cemento (CSM). Se incorporaron tres tamaños diferentes de partículas de caucho en migajas (malla 40, malla 60 y malla 80) en CSM con tres contenidos diferentes (sustituyendo $1.0 \%, 1.5 \%$ y $2.0 \%$ de agregados finos por volumen). Los resultados indicaron que, debido a la elasticidad de las partículas de caucho, la resistencia a la compresión del CSM disminuyó con el aumento del contenido de caucho.

\section{e) Durabilidad}

(Gupta, Siddique, Sharma, \& Chaudhary, 2019), estudiaron la durabilidad del (polvo de caucho y fibras de caucho). Los resultados mostraron que, a pesar de mostrar una buena resistencia contra el ataque de ácido y la difusión de iones de cloruro, la barra de refuerzo incrustada en concreto con caucho era muy propensa a la corrosión en comparación con la barra de refuerzo en el concreto de control.

(Guo, Dai, Si, Sun, \& Lu, 2017), utilizaron dos métodos de tratamiento de superficies ( $\mathrm{NaOH}$ y agente de acoplamiento de silano) y tres técnicas de recubrimiento (recubierto con cemento normal, cemento mezclado con humo de sílice y cemento mezclado más silicato de sodio) para mejorar la unión caucho-cemento. En general este estudio demostró que los métodos tratados con $\mathrm{NaOH}$ pueden mejorar el rendimiento mecánico del concreto con caucho y mejorar su durabilidad a largo plazo

(Miller \& Tehrani, 2017), realizaron una investigación detallada del concreto con caucho utilizando 38 muestras cilíndricas y 36 de viga. Seis diseños de mezcla, incorporados en el estudio, contenían relaciones de reemplazo de caucho de $0 \%$ a $100 \%$ por volumen de reemplazo de un agregado grueso de lutita expandida liviana expandida. Los resultados sugieren que los agregados derivados de neumáticos reducen la resistencia mecánica de las muestras, pero mejoran la ductilidad y dureza de los materiales.

(Zhu et al., 2018), en su investigación demostraron que la adición de caucho desmenuzado puede aumentar la saturación capilar del concreto y reducir el grado de corrosión de las barras de acero en el concreto, y con el aumento del contenido de caucho desmenuzado, la tasa de pérdida de peso de 
las barras de acero disminuye. Se muestra que el concreto con caucho granulado tiene una durabilidad diferente con la temperatura ambiente de prueba y puede proporcionar una referencia para la aplicación del concreto de caucho granulado en la penetración de iones anti cloruro.

(Shahjalal et al, 2020), en su estudio están buscando formas de reducir y reutilizar estos materiales de desecho provenientes de neumáticos para promover una práctica de construcción sostenible y una de las formas es incorporar caucho fino proveniente de los neumáticos desechados y agregado grueso en la producción de concreto pertenece a un futuro sostenible de la industria de la construcción. Se encuentra que los valores de tenacidad y ductilidad aumentan para el contenido de caucho hasta un 5\% y luego comienzan a disminuir para el $10 \%$ de caucho desmenuzado.

\section{f) Tenacidad:}

(Eisa, Elshazli, \& Nawar, 2020), experimentaron el efecto de las fibras de caucho y acero desmenuzado en las vigas RC. Incorporando 10\%, el caucho mejoró la ductilidad y la tenacidad de las mezclas de concreto. Combinando $1 \%$ de fibras de acero y $10 \%$, el caucho mejoró el rendimiento de las vigas RC. Los resultados de las pruebas mostraron que el uso de caucho fino como reemplazo parcial de los agregados finos en un $5 \%$ y $10 \%$ muestra buenas propiedades mecánicas con un aumento de la tenacidad en un $6 \%$ y $5 \%$, respectivamente, y un aumento en la

mejora el rendimiento de la tenacidad en un $3 \%$, aumento de la resistencia a la compresión en un $11 \%$, aumento de la ductilidad en un $3 \%$, aumento del módulo de elasticidad en un $3 \%$ en las vigas de concreto armado con porcentajes de caucho superiores al $10 \%$.
(Mo et al., 2020), en su estudio agregaron un $4.5 \%$ de polvo de caucho para mejorar las propiedades de amortiguación del concreto en emulsión de estireno-acrílico. Los resultados experimentales muestran que la adición de emulsión de estireno-acrílico mejora la tenacidad y la capacidad de amortiguación del concreto en aproximadamente un 20,4\%. pero reduce la resistencia a la compresión en aproximadamente un $36 \%$. El polvo de caucho reduce la resistencia a la compresión en aproximadamente un 21,4\% y acelera la degradación de la rigidez dinámica al tiempo que mejora la capacidad de amortiguación del concreto en aproximadamente un $20,4 \%$.

(Abbassi \& Ahmad, 2020), estableció una investigación experimental para comprender el comportamiento de compresión del concreto mediante la sustitución de agregados naturales con caucho de neumáticos desechados en diferentes porcentajes, que van del $10 \%$ al $50 \%$ en volumen. Los resultados experimentales indicaron que el comportamiento del concreto cambió de frágil a dúctil y su capacidad para absorber energía de compresión (tenacidad a la compresión) mejoró al aumentar el porcentaje de caucho desmenuzado.

\section{5.- Propiedades mejoradas del concreto}

\section{a) Conductividad térmica:}

Los investigadores descubrieron que la inclusión de caucho fino en un $10 \%$ da como resultado una mejora del aislamiento térmico del $23 \%$. Estos estudios han demostrado que el concreto recubierto de goma que contiene partículas de caucho desmenuzado tiene una conductividad térmica más baja en comparación con el concreto convencional (Mushunje, Otieno, \& Ballim, 2018).

(Abd-Elaal et al., 2019), en su estudio presenta un enfoque novedoso para tratamientos previos de partículas de 
caucho granulado mediante un tratamiento térmico a $200{ }^{\circ} \mathrm{C}$ antes de su incorporación al concreto. El tiempo de calentamiento, el tamaño del caucho y el contenido de caucho fueron las variables en esta investigación experimental. Los resultados mostraron mejoras prometedoras en el rendimiento del concreto en comparación con los resultados de trabajos anteriores. Con un contenido de caucho del $20 \%$ utilizando caucho tratado térmicamente de malla de tamaño \# 40, la resistencia a la compresión se recuperó en un $60,3 \%$.

(Tang et al, 2020), investigaron las propiedades compresivas de concreto agregado reciclado modificado con caucho RRAC después de la exposición a temperaturas elevadas. Se estudiaron la curva tensión-deformación, la resistencia a la compresión, el rendimiento de deformación y la resistencia al estallido de muestras de RRAC con contenidos de caucho de $0 \%, 4 \%$ y $9 \%$ después de la exposición a 20, 200, 400 y $600{ }^{\circ} \mathrm{C}$ durante 60 minutos. En conclusión, una proporción de aproximadamente $4 \%$ presentó el mejor desempeño de resistencia al fuego bajo cargas de compresión. RRAC puede reemplazar al RAC y es apropiado para su uso en estructuras que requieren resistencia al calor,

(Záleská, Pavlík, Cítek, Jankovsky, \& Pavlíková, 2019), investigaronlas propiedades del concreto después de su exposición térmica incluyó pruebas de pérdida de masa, resistencia mecánica, densidad aparente y conductividad térmica que se probó como propiedad que tiene relación directa con los parámetros estructurales del concreto. El uso de agregado de caucho de neumáticos resultó en una disminución del peso unitario, un empeoramiento de los parámetros mecánicos y una reducción significativa de la conductividad térmica del concreto. El agregado a base de caucho casi no afectó las propiedades de transporte de agua, mientras que las propiedades de transporte de vapor de agua aumentaron con la cantidad de caucho en la mezcla.

(Akbarzadeh Bengar, Shahmansouri, Akkas Zangebari Sabet, Kabirifar, \& WY Tam, 2020), investigaron a fondo el comportamiento mecánico del concreto de caucho tras un incendio. Se probó el efecto simultáneo del fuego y la adición de agregado de caucho sobre el concreto, reemplazando el agregado fino en un 5, 10, 15 y $20 \%$, bajo altas temperaturas de 200, 400, 600 y 800 ${ }^{\circ} \mathrm{C}$. Se presentaron modelos predictivos de propiedades estructurales de RRC bajo fuego. Los resultados indican un notable deterioro de las características físico-mecánicas de las probetas de concreto a medida que aumenta la temperatura.

(Alvarez, Gutierrez, Duran, \& Pacheco, 2020), buscaron evaluar una mayor resistencia al corte y capacidad de soporte para la penetración del suelo arcilloso mediante la adición de $1.5 \%, 2.5 \%$ y $3.5 \%$ de caucho en polvo. Para esto, se realizó el análisis de límites de Atterberg, la prueba de compactación del supervisor modificado, la caja de corte y el CBR. Los resultados reflejan que la cohesión de la mezcla aumentó y el ángulo de fricción interna disminuyó con respecto al suelo natural, resultando en la suma de un aumento de la resistencia al corte.

(Khern et al., 2020), en su estudio demostraron que, con la incorporación de caucho proveniente de neumáticos, reduce la resistencia, aumenta la permeabilidad $\mathrm{y}$ disminuye la conductividad térmica del concreto. Sin embargo, solo unos pocos estudios han investigado el efecto de los agregados de caucho de superficie modificada sobre las propiedades del concreto.

\section{b) Propiedades acústicas:}

El concreto recubierto de caucho es efectivo para absorber energía de sonido, impacto y vibraciones en comparación con el concreto 
simple. Los investigadores encontraron que la absorción de sonido y el coeficiente de reducción de ruido aumentaron con un aumento en los niveles de reemplazo de más de $20 \%$ de caucho desmenuzado. Por lo tanto, sugirieron que la mayor absorción acústica del concreto recubierto de caucho se debe al aumento de los huecos de aire en el concreto (Mushunje, Otieno, \& Ballim, 2018).

(Wang \& Du, 2020), consideraron el tamaño de partícula, la composición y la tasa de sustitución del material de caucho; se compararon y analizaron el rendimiento físico, el rendimiento térmico y el rendimiento acústico del concreto normal, el concreto de agregado reciclado y el concreto de caucho granulado reciclado RCC. Se compararon los principales índices de diferentes concretos, incluyendo densidad aparente, porosidad, coeficiente de transferencia de calor, resistencia térmica, coeficiente de absorción acústica y coeficiente de reducción de ruido. Los resultaos demuestran que, con el aumento de la tasa de reemplazo de caucho reciclado, la densidad aparente de la serie RCC disminuye gradualmente, oscilando entre 1700 y 2100 $\mathrm{kg} / \mathrm{m} \mathrm{3}$, mientras que la densidad aparente de NC y RC es $2390 \mathrm{~kg} / \mathrm{m} 3$ y $2360 \mathrm{~kg} / \mathrm{m}$ 3. Cuando la tasa de reemplazo de agregado reciclado es del $20 \%$ y la tasa de reemplazo del material de caucho es del 10\% - 30\%.

\section{c) Conductividad hidráulica:}

La conductividad hidráulica es una propiedad fundamental del material que debe tenerse en cuenta al determinar la idoneidad de un material para su uso en sistemas de drenaje. Por tanto, se han realizado estudios con el objetivo de determinar la conductividad hidráulica de los (productos derivados de neumáticos) TDP. Para ser un componente exitoso en los sistemas de drenaje, los TDP deben permitir que la humedad se disipe a un ritmo deseable. Aunque la inclusión de TDP en los sistemas de drenaje puede proporcionar mejoras en la permeabilidad, porosidad y absorción sobre los agregados tradicionales, se debe considerar el lixiviado potencial resultante de la saturación continua del concreto (Mohajerani et al., 2020).

(Jasim \& Abdulabbas, 2020), presentaron información experimental sobre concreto permeable con sustitución fraccionada de agregado grueso por caucho de neumáticos desechados. La grava natural se sustituyó mediante el método volumétrico por reemplazos de $0,10,20,30,40$ y $50 \%$ para los distintos.

\section{d) Carbonatación.}

(Vilches, Figueroa, Chamorro, \& Piña, 2020), analizaron el efecto de la carbonatación en el concreto con agregado de caucho proveniente de neumáticos como un reemplazo proporcional del $0 \%, 10 \%, 20 \%, 30 \%, 40 \%$, $50 \%$ y $60 \%$ del agregado fino. La prueba de carbonatación se realizó durante $2,4,6$ y 24 horas. La resistencia a la compresión del concreto disminuyó dependiendo del contenido de caucho de neumáticos en las mezclas, mientras que el concreto con porcentajes de caucho de neumáticos superiores al $30 \%$ se vieron afectados por la carbonatación.

\section{CONCLUSIONES}

La presencia de partículas de caucho provenientes de neumáticos y usados en el concreto, sustituyendo a los agregados convencionales ha llamado recientemente la atención de los investigadores a nivel mundial a tener más interés por estudiar los efectos de las partículas de caucho en la mezcla de concreto. En este sentido, la mayoría de los investigadores han sugerido un contenido máximo de caucho de no más del $20 \%$ del volumen total agregado y un tamaño no mayor que el tamaño del caucho desmenuzado. 
Desde una perspectiva de resistencia a la compresión a los 28 días, la mayoría de investigadores llegan a la conclusión que el concreto con caucho se puede utilizar, pero con proporciones de 0 a $12.5 \%$ en peso sustituyendo al agregado fino para obtener concretos de alta resistencia con un límite de $60 \mathrm{MPa}$, luego empieza a bajar su resistencia si aumentamos las proporciones. Por otro lado, se reduce según la resistencia a la flexión en un $12.8 \%$ cuando se sustituye el $20 \%$ de agregado fino por agregado de caucho.

El costo de procesamiento y trituración de los neumáticos depende del tamaño de la partícula, es decir si las partículas de caucho son menores, entonces el costo asociado con su producción será mucho mayor.

Es evidente que a partir de las investigaciones con el uso de caucho en el concreto es una opción viable $\mathrm{y}$ fomenta el concepto de sostenibilidad y desarrollo de estructuras ecológicas. Además, la baja densidad del concreto con caucho se puede utilizar en el desarrollo de concreto ligero para lograr diseños más económicos.

Aunque se ha trabajado mucho para comprender mejor los efectos del caucho proveniente de neumáticos en las propiedades del concreto, todavía se necesitan más investigaciones, en particular, carbonatación y durabilidad del cloruro.

\section{REFERENCIAS BIBLIOGRÁFICAS}

Abbassi, F., \& Ahmad, F. (2020). Behavior analysis of concrete with recycled tire rubber as aggregate using 3D-digital image correlation. Journal of Cleaner Production, 274, 1-12. doi:https://doi. org/10.1016/j.jclepro.2020.123074

Abd-Elaal, E.-S., Araby, S., Mills, J. E., Youssf, O., Roychand, R., Ma, X., . . . Gravina, R. J. (2019).
Novel approach to improve crumb rubber concrete strength using thermal treatment. Construction and Building Materials, 229, 1-11. doi:https://doi.org/10.1016/j. conbuildmat.2019.116901

Abdelmonem, A., El-Feky M, S., Nasr, E.-S. A., \& Kohail, M. (2019). Performance of high strength concrete containing recycled rubber. Construction and Building Materials, 227, 1-10. doi:https://doi.org/10.1016/j. conbuildmat.2019.08.041

Adeboje, A., Kupolati, W., Sadiku, E., \& Ndambuki, J. (2020). Characterization of Modified Crumb Rubber Concrete. International Journal of Sustainable Development and Planning, 15(3), 377-383. doi:https://doi. org/10.18280/ijsdp.150315

Akbarzadeh Bengar, H., Shahmansouri, A. A., Akkas Zangebari Sabet, N., Kabirifar, K., \& WY Tam, V. (2020). Impact of elevated temperatures on the structural performance of recycled rubber concrete: Experimental and mathematical modeling. Construction and Building Materials, 255, 1-15. doi:https://doi.org/10.1016/j. conbuildmat.2020.119374

Al Rawahi, Z., \& Bilal Waris, M. (2017). Use of recycled tires in nonstructural concrete. MATEC Web of Conferences(120), 1-9. doi:https://doi.org/10.1051/ matecconf $/ 201712003002$

Alvarez, N., Gutierrez, J., Duran, G., \& Pacheco, L. (2020). Experimental study of the mechanical effect of a clayey soil by adding rubber powder for geotechnical applications. IOP Conference Series: Materials Science and Engineering, 758. doi:10.1088/ 1757-899X / 758/1/012057

Bandarage, K., \& Sadeghian, P. (2019). Effects of long shredded rubber particles recycled from waste tires on mechanical properties of concrete. Journal of Sustainable Cement-Based Materials, 9(1), 1-11. doi:https://doi. 
org/10.1080/21650373.2019.167683 9

Banerjee, S., Mandal, A., \& Dr. Jessy Rooby, J. (2020). REVIEW OF TYRE AS AGGREGATE REPLACEMENT IN CONCRETE. Journal of Critical Reviews, 7(9), 994-996. doi:http:// dx.doi.org/10.31838/jcr.07.09.183

Bisht, K., \& Ramana, P. V. (2017). Evaluation of mechanical and durability properties of crumb rubber concrete. Construction and Building Materials, 155, 811817. doi:https://doi.org/10.1016/j. conbuildmat.2017.08.131

BušiC, R., BenšiC, M., MiliceviC, I., \& Strukar, K. (Abr de 2020). Prediction Models for the Mechanical Properties of Self-Compacting Concrete with Recycled Rubber and Silica Fume. Materials, 13(8), 1-25. doi:10.3390/ ma13081821

Choudhary, S., Chaudhary, S., Jain, A., \& Gupta, R. (2020). Assessment of effect of rubber tyre fiber on functionally graded concrete. Materials Today: Proceedings, 28, 1496-1502. doi:https://doi.org/10.1016/j. matpr.2020.04.830

Chylík, R., Trtík, T., Fládr, J., \& Bílý, P. (2017). Mechanical properties and durability of crumb rubber concrete. IOP Conference Series: Materials Science and Engineering, 236, 1-7. doi:10.1088 / 1757-899X / 236/1/012093

Eisa, A. S., Elshazli, M. T., \& Nawar, M. T. (2020). Experimental investigation on the effect of using crumb rubber and steel fibers on the structural behavior of reinforced concrete beams. Construction and Building Materials, 252, 1-13. doi:https://doi.org/10.1016/j. conbuildmat.2020.119078

Eltayeb, E., Ma, X., Zhuge, Y., Youssf, O., \& Mills, J. E. (2020). Influence of rubber particles on the properties of foam concrete. Journal of Building Engineering, 30, 1-13. doi:https://doi. org/10.1016/j.jobe.2020.101217

Gerges, N. N., Issa, C. A., \& Fawaz, S. A. (2018). Rubber concrete: Mechanical and dynamical properties. Case Studies in Construction Materials, 9, 1-17. doi:https://doi.org/10.1016/j. cscm.2018.e00184

Guo, S., Dai, Q., Si, R., Sun, X., \& Lu, C. (2017). Evaluation of properties and performance of rubber-modified concrete for recycling of waste scrap tire. Journal of Cleaner Production, 148, 681-689. doi:https://doi. org/10.1016/j.jclepro.2017.02.046

Gupta, T., Siddique, S., Sharma, R. K., \& Chaudhary, S. (2019). Behaviour of waste rubber powder and hybrid rubber concrete in aggressive environment. Construction and Building Materials, 217, 283291. doi:https://doi.org/10.1016/j. conbuildmat.2019.05.080

Huang, W., Huang, X., Xing, Q., \& Zhou, Z. (2020). Strength reduction factor of crumb rubber as fine aggregate replacement in concrete. Journal of Building Engineering, 32, 1-6. doi:https://doi.org/10.1016/j. jobe. 2020.101346

Irmawaty, R., Parung, H., \& Md Noor, N. (2020). Experimental study of rubber particles from recycle tires as concrete. IOP Conference Series: Earth and Environmental Science, 473, págs. 1-7. doi:10.1088/17551315/473/1/012130

Jalal, M., Nassir, N., Jalal, H., \& Arabali, P. (2019). On the strength and pulse velocity of rubberized concrete containing silica fume and zeolite: Prediction using multivariable regression models. Construction and Building Materials, 223, 530543. doi:https://doi.org/10.1016/j. conbuildmat.2019.06.233

Jasim, A. T., \& Abdulabbas, Z. H. (2020). Production of sustainable pervious concrete by using waste tires rubber as partial replacement of coarse aggregate. 2ND 
INTERNATIONAL CONFERENCE ON MATERIALS ENGINEERING \& SCIENCE (ICONMEAS 2019), (págs. 1-7). doi:https://doi. org/10.1063/5.0000255

Khern, Y. C., Paul, S. C., Kong, S. Y., Babafemi, A. J., Anggraini, V., Miah, M. J., \& Šavija, B. (2020). Impact of Chemically Treated Waste Rubber Tire Aggregates on Mechanical, Durability and Thermal Properties of Concrete. frontiers in Materials, 7, 1-11. doi:https://doi.org/10.3389/ fmats. 2020.00090

Lei, B., Liu, H., Yao, Z., \& Tang, Z. (2019). Experimental study on the compressive strength, damping and interfacial transition zone properties of modified recycled aggregate concrete. Royal Society Open Science, 6(12), 1-14. doi: http://dx.doi.org/10.1098/ rsos. 190813

Li, Y., Zhang, S., Wang, R., \& Dang, F. (2019). Potential use of waste tire rubber as aggregate in cement concrete - A comprehensive review. Construction and Building Materials, 225, 11831201. doi:https://doi.org/10.1016/j. conbuildmat.2019.07.198

Lv, J., Du, Q., Zhou, T., He, Z., \& Li, K. (2019). Fresh and Mechanical Properties of Self-Compacting Rubber Lightweight Aggregate Concrete and Corresponding Mortar. Avances en Ciencia de Materiales e Ingeniería, 2019, 1-14. doi:https:// doi.org/10.1155/2019/8372547

Medina, N. F., Medina, D. F., HernándezOlivares, F., \& Navacerrada, M. (2017). Mechanical and thermal properties of concrete incorporating rubber and fibres from tyre recycling. Construction and Building Materials, 144, 563573. doi:https://doi.org/10.1016/j. conbuildmat.2017.03.196

Miller, N. M., \& Tehrani, F. M. (2017). Mechanical properties of rubberized lightweight aggregate concrete. Construction and
Building Materials, $147, \quad 264-$ 271. doi:https://doi.org/10.1016/j. conbuildmat.2017.04.155

Mo, J., Zeng, L., Guo, F., Liu, Y., Ma, L., Chen, B., \& Liu, X. (2020). Experimental study on damping properties of rubber powder modified styreneacrylic emulsion concrete beam. Journal of Building Engineering, 32, 1-11. doi:https://doi.org/10.1016/j. jobe. 2020.101728

Mohajerani, A., Burnett, L., Smith, J. V., Markovski, S., Rodwell, G., Rahman, M. T., . . Maghool, F. (2020). Recycling waste rubber tyres in construction materials and associated environmental considerations: A review. Resources, Conservation \& Recycling, 155, 1-17. doi:https://doi. org/10.1016/j.resconrec.2020.104679

Mousavimehr, M., \& Nematzadeh, M. (2019). Predicting postfire behavior of crumb rubber aggregate concrete. Construction and Building Materials, 229, 1-17. doi:https://doi.org/10.1016/j. conbuildmat.2019.116834

Mushunje, K., Otieno, M., \& Ballim, Y. (2018). A review of Waste Tyre Rubber as an Alternative Concrete Consituent Material. MATEC Web of Conferences , 199, 1-8. doi:https://doi.org/10.1051/ matecconf/201819911003

Muttashar, M., Alomari, K., \& Al-Umar, M. (2020). Influence of Waste Rubbers Particle Size as Partial Substitution with Coarse Aggregate on Compressive property and water absorption ratio of Concrete. IOP Conference Series: Materials Science and Engineering, 870, 1-6. doi:https://doi.org/10.1088/1757899X/870/1/012104

Najmi, A. M., Mariyana, A. K., Shek, P. N., \& Nurizaty, Z. .. (2020). Hardened properties of concrete with different proportion of crumb rubber and fly ash. IOP Conference Series: Materials Science and Engineering, 849, págs. 1-10. Malasia. doi:10.1088 


\section{/ 1757-899X / 849/1/012038}

Roychand, R., Gravina, R. J., Zhuge, Y., Ma, X., Youssf, O., \& Mills, J. E. (2019). A comprehensive review on the mechanical properties of waste tire rubber concrete. Construction and Building Materials, 237, 1-20. doi:https://doi.org/10.1016/j. conbuildmat.2019.117651

Senin, M. S., Shahidan, S., Leman, A. S., Othman, N., Shamsuddin, S.m., MHW Ibrahim, M., \& Mohd Zuki, S. (2017). The durability of concrete containing recycled tyres as a partial replacement of fine aggregate. IOP Conference Series: Materials Science and Engineering, 271, 1-7. doi:10.1088/1757$899 X / 271 / 1 / 012075$

Shahjalal, M., Islam, K., Rahman, J., Ahmed, K. S., Karim, M. R., \& Billah, A. H. (2020). Flexural response of fiber reinforced concrete beams with waste tires rubber and recycled aggregate. Journal of Cleaner Production, 278, 1-18. doi:https://doi.org/10.1016/j. jclepro.2020.123842

Shao, J., Zhu, H., Zuo, X., Lei, W., Borito, S. M., Liang, J., \& Duan, F. (2020). Effect of waste rubber particles on the mechanical performance and deformation properties of epoxy concrete for repair. Construction and Building Materials, 241, 1-12. doi:https://doi.org/10.1016/j. conbuildmat.2020.118008

Si, R., Guo, S., \& Dai, Q. (2017). Durability performance of rubberized mortar and concrete with $\mathrm{NaOH}-\mathrm{Solution}$ treated rubber particles. Construction and Building Materials, 153, 496505. doi:https://doi.org/10.1016/j. conbuildmat.2017.07.085

Silva, F. M., Miranda Jr., E. J., Dos Santos, J. M., Gachet-Barbosa, L. A., Gomes, A. E., \& Lintz, R. C. (2019). The use of tire rubber in the production of high-performance concrete. ceramica, 65(suppl 1), 110-114. doi:https://doi.org/10.1590/0366-

\section{$6913201965 \mathrm{~s} 12598$}

Sun, X., Wu, S., Yang, J., \& Yang, R. (2020). Mechanical properties and crack resistance of crumb rubber modified cement-stabilized macadam. Construction and Building Materials, 259, 1-8. doi:https://doi.org/10.1016/j. conbuildmat.2020.119708

Svoboda, J., Václavík, V., Dvorský, T., Klus, L., \& Botula, J. (2020). The Utilization of a Combination of Recycled Rubber from Waste Tires and Waste Waters from a Concrete Plant in the Production of Cement Composites. Key Engineering Materials, 838, 5966. doi:https://doi.org/10.4028/www. scientific.net/KEM.838.59

Tang, Y., Feng, W., Feng, W., Chen, J., Bao, D., \& Li, L. (2020). Compressive properties of rubber-modified recycled aggregate concrete subjected to elevated temperatures. Construction and Building Materials, 1-11. doi:https://doi.org/10.1016/j. conbuildmat.2020.121181

Vilches, J., Figueroa, J., Chamorro, L., \& Piña, C. (2020). Analysis of the Carbonation in Normal Concrete with the Addition of Tire Rubber Aggregate. IOP Conference Series: Earth and Environmental Science, 503, págs. 1-10. doi:10.1088/17551315/503/1/012069

Wang, J., \& Du, B. (2020). Experimental studies of thermal and acoustic properties of recycled aggregate crumb rubber concrete. Journal of Building Engineering, 32, 1-7. doi:https://doi. org/10.1016/j.jobe.2020.101836

XU, J., Yao, Z., Yang, G., \& Han, Q. (2020). Research on crumb rubber concrete: From a multiscale review. Construction and Building Materials, 232, 1-25. doi:https://doi.org/10.1016/j. conbuildmat.2019.117282

Yang, G., Chen, X., Xuan, W., \& Chen, Y. (2018). Dynamic compressive and splitting tensile properties of concrete containing recycled tyre rubber under 
high strain rates. Sädhanā, 43(11), 1-13. doi:https://doi.org/10.1007/ s12046-018-0944-5

Youssf, O., Mills, J. E., Benn, T., Zhuge, Y., Ma, X., Roychand, R., \& Gravina, R. (2020).DevelopmentofCrumbRubber Concrete for Practical Application in the Residential Construction Sector Design and Processing. Construction and Building Materials, 260, 1-12. doi:https://doi.org/10.1016/j. conbuildmat.2020.119813

Záleská, M., Pavlík, Z., Cítek, D., Jankovsky, O., \& Pavlíková, M. (2019). Ecofriendly concrete with scrap-tyrerubber-based aggregate - Properties and thermal stability. Building and construction materials, 225, 709722. doi:https://doi.org/10.1016/j. conbuildmat.2019.07.168

Zhu, H., Liang, J., Xu, J., Bo, M., Li, J., \& Tang, B. (2018). Research on antichloride ion penetration property of crumb rubber concrete at different ambient temperatures. Construction and Building Materials, 189, 4253. doi:https://doi.org/10.1016/j. conbuildmat.2018.08.1 